Voix et Images

volxetimages

\title{
L'histoire dans les romans de Laure Conan. Lecture sémiotique de l'idéologie de la langue gardienne de la foi
}

\section{Fernand Roy}

Volume 25, numéro 2 (74), hiver 2000

Le champ littéraire de la jeunesse au carrefour de la recherche universitaire

URI : https://id.erudit.org/iderudit/201484ar

DOI : https://doi.org/10.7202/201484ar

Aller au sommaire du numéro

\section{Éditeur(s)}

Université du Québec à Montréal

\section{ISSN}

0318-9201 (imprimé)

1705-933X (numérique)

Découvrir la revue

Citer cet article

Roy, F. (2000). L'histoire dans les romans de Laure Conan. Lecture sémiotique de l’idéologie de la langue gardienne de la foi. Voix et Images, 25(2), 328-348. https://doi.org/10.7202/201484ar
Résumé de l'article

À l'encontre de la thèse de l'influence abusive de l'abbé Henri-Raymond Casgrain sur Laure Conan, il s'avère que l'instance d'énonciation d'Angéline de Montbrun pratiquait déjà spontanément une écriture orientée vers la valorisation de l'histoire. En se fondant sur une analyse sémiotique de la narration et de renonciation des romans historiques de la première romancière du Québec, le présent article propose l'hypothèse suivante : un roman argumente en vue de l'attribution (par le lecteur) d'une valeur ajoutée au fait de raconter (par écrit). La stratégie enonciative consiste à appeler la valorisation de l'histoire - liée à l'écriture - au moyen de la mise en oeuvre d'une interaction verbale fictive. L'article en arrive à la conclusion que si, comme le prétend l'idéologie libérale depuis I960, ces romans historiques relèvent de notre préhistoire, c'est parce qu'ils faisaient une équation d'identité entre littérarité et historicité. De la littérature comme constitution du " patrimoine sacré de la langue française » à celle qui se fait moderne en faisant le pari d'un " pays à inventer " d'abord dans les mots, il y aurait simplement prise de conscience de ce que l'histoire est à faire - et affaire d'écriture. Rien ne servirait d'essayer, en retour, d'établir une équation d'identité entre historicité et littérarité. 


\title{
L'histoire dans les romans de Laure Conan. Lecture sémiotique de l'idéologie de la langue gardienne de la foi
}

\author{
Fernand Roy, Université du Québec à Chicoutimi
}

\begin{abstract}
À l'encontre de la thèse de l'influence abusive de l'abbé Henri-Raymond Casgrain sur Laure Conan, il s'avère que l'instance d'ênonciation d'Angéline de Montbrun pratiquait déjà spontanément une écriture orientée vers la valorisation de l'bistoire. En se fondant sur une analyse sémiotique de la narration et de l'énonciation des romans bistoriques de la première romancière du Québec, le présent article propose l'bypothèse suivante: un roman argumente en vue de l'attribution (par le lecteur) d'une valeur ajoutée au fait de raconter (par écrit). La stratégie énonciative consiste à appeler la valorisation de l'bistoire - liée à l'écriture - au moyen de la mise en ouvre d'une interaction verbale fictive. L'article en arrive à la conclusion que si, comme le prétend l'idéologie libérale depuis 1960, ces romans historiques relèvent de notre prébistoire, c'est parce qu'ils faisaient une équation d'identité entre littérarité et historicité. De la littérature comme constitution du "patrimoine sacré de la langue française "à celle qui se fait moderne en faisant le pari d'un "pays à inventer" d'abord dans les mots, il y aurait simplement prise de conscience de ce que l'bistoire est à faire - et affaire d'écriture. Rien ne servirait d'essayer, en retour, d'établir une équation d'identité entre bistoricité et littérarité.
\end{abstract}

Gilles Marcotte a écrit que "le premier personnage né au Canada français s'appel[ait] Angéline de Montbrun ${ }^{1}$ "; et que Laure Conan "enrichi[ssait] sa vision de l'histoire d'une expérience toute personnelle de la misère ${ }^{2}$. Sa pensée au sujet de notre première romancière recoupe, d'une part, celle de notre premier historien de la littérature, Camille Roy, pour qui "ce que Laure Conan aime surtout faire en ses livres, c'est de la psychologie ${ }^{3}$ " et, d'autre part, celle du directeur du DOLQ, Maurice

1. Gilles Marcotte, Une littérature qui se fait, Montréal, HMH, 1962, p. 16.

2. Ibid., p. 18.

3. Camille Roy, Romanciers de chez nous, Montréal, Beauchemin, 1935, p. 113. 
Lemire, selon qui, "sous la férule de l'abbé Casgrain [Laure Conan dut] se consacrer au roman historique ${ }^{4}$, avec comme résultat que pour écrire À l'ouvre et à l'épreuve elle aurait "cherché dans l'histoire une aventure semblable à la sienne ${ }^{5}$. Ces trois historiens majeurs partagent l'idée suivante: dans la perspective d'une constitution progressive de la littérature [d'abord dite canadienne-française puis] québécoise, l'originalité du travail littéraire de Laure Conan tiendrait au fait qu'elle a donné une psychologie aux personnages romanesques "nés" au Canada français. Et cette idée en sous-tend une autre, celle que notre première romancière ait eu recours, un peu malgré elle, au genre "historique".

La thèse sous-jacente prétend que Laure Conan aurait été manipulée par l'abbé Henri-Raymond Casgrain; elle permet de voir dans Angéline de Montbrun la première manifestation du roman moderne (entendre psychologique). Cette thèse "individualiste-bourgeoise" présente une faille importante: on peut se demander si elle n'occulte pas, à travers le rejet de la vision "cléricalo-religieuse" de l'histoire que privilégiait l'abbé Casgrain, la nécessité de penser la fonction de l'histoire dans la constitution d'une littérature. En renvoyant tout ce qui n'est pas perçu comme "moderne" du côté d'une sorte de "prélittérature" — sinon d'une sorte de préhistoire ne néglige-t-elle pas de penser objectivement le lien entre "histoire" et "littérature"? En tout cas, on ne saurait admettre que l'argument ultime soit dans le mot "liberté": c'est là une notion qui, d'évidence, est liée à la vision de l'histoire-progrès que véhicule toute histoire littéraire d'inspiration libérale. La notion gagnerait à tout le moins à être définie en relation avec l'activité d'écriture même: faut-il concevoir la liberté par l'écriture comme liée à l'absence immédiate d'interlocuteur? absence qui, au-delà du "traumatisme de la page blanche", fait qu'un écrivant se libère momentanément et fictivement de la loi de la pesanteur des systèmes langagiers et peut avoir l'impression qu'il crée individuellement du sens?

Aussi, dans une perspective sémiotique, en partant du postulat que la vision libérale du littéraire doit être décodée au même titre que la vision cléricalo-religieuse contre laquelle elle s'est développée, il m'a semblé pertinent de revenir sur l'hypothèse de la rupture dans l'œuvre de Laure Conan: serait-il possible que la vision de la littérature qu'a cru imposer l'abbé Casgrain à notre première romancière ait déjà été en germe dans Angéline de Montbrun? Se pourrait-il que Casgrain ait seulement contribué à accentuer une vision de l'écriture qui était déjà inscrite dans le premier récit de Laure Conan?

L'héroïne d'Angéline de Montbrun était déjà lectrice de l'Histoire de Garneau avant la célèbre préface de Casgrain à la seconde publication [en

\footnotetext{
4. Maurice Lemire (dir.), Dictionnaire des seuvres littéraires du Québec, tome 1, Montréal, Fides, 1978, p. xxxi.

5. Ibid., p. 10.
} 
un livre autonome] du roman, qui avait été accueillie favorablement par les gens de l'École patriotique de Québec. Et une analyse sémio-narrative du roman ${ }^{6}$ conduit à suggérer que l'influence, présumée néfaste, de Casgrain sur Laure Conan a peut-être été mal identifiée, sinon carrément exagérée $^{7}$ : si le "premier personnage romanesque né au Canada français" parvient à sublimer sa déception amoureuse dans l'écriture intime, il ne faut pas pour autant oublier que l'amorce de sa "thérapie d'écriture" tient à ce qu'il tire profit; et leçon, à la fois de l'expérience d'écriture de sa mère et de celle de l'historien F.-X. Garneau. Il convient ici d'aller lire le roman de près, pour établir que l'activité d'écriture de ce "premier personnage romanesque " est très étroitement liée à l'énonciation du texte même.

Dans le bref récit au centre du roman, entre la première partie épistolaire et la dernière partie, "Feuilles détachées", l'instance d'énonciation invite expressément le lecteur - un public possible, en l'occurrence formé des personnes qui ont aimé et qui ont souffert - à parcourir le journal écrit par l'héroïne après la mort de son père et la défection de son fiancé; et elle le fait en réutilisant les termes déjà employés pour désigner, dans une lettre de la première partie, le journal intime de la mère d'Angéline. Je cite d'abord le passage de la lettre où Mina apprenait à son frère Maurice (le fiancé en question) ce qu'Angéline lui avait déjà confié au sujet du journal intime de sa mère, puis le passage du récit central où est présentée au lecteur la section "Feuilles détachées ${ }^{8}$ " qui reproduit le journal d'Angéline :

Angéline m'a raconté que le jour de ses noces, son père alla à son travail. Oui, mon cher - c'est écrit dans quelques pages intimes que Madame de Montbrun a laissées - dans la matinée il s'en fut à ses champs. ( $A M, 16$. C'est moi qui souligne.)

Cette noble jeune fille, qui s'isolait dans la douleur, avec la fière pudeur des âmes délicates, écrivait un peu quelquefois. Ces pages intimes intéresseront peut-être ceux qui ont aimé et souffert. ( $A M, 70$. C'est moi qui souligne.)

Des pages intimes et demeurées "privées" de la mère aux pages intimes de sa fille qui sont jugées dignes d'une lecture publique, il y a que, en plus d'avoir été éprouvée plus radicalement, la seconde peut compter sur l'exemple que constitue désormais l'bistorien Garneau:

6. Analyse dont l'essentiel a été intégré à un article: Louise Milot et Fernand Roy, "On Textual Reference to Writing and its Correlation with Literary History", Poetics Today, vol. XII, n 4 , p. 713-728.

7. Voir notre introduction au roman dans l'édition de 1991 de la Bibliothèque québécoise.

8. Les références aux quatre romans de Laure Conan seront indiquées entre parenthèses, à la suite des citations, à l'aide des sigles $A M$ (Angéline de Montbrun, Montréal, Bibliothèque québécoise, 1991), $C E$ (Á l'œuvre et à l'épreuve, Montréal, Beauchemin, 1958), O (L'oublié, Montréal, Beauchemin, 1902), et SI (La sève immortelle, Montréal, Bibliothèque de l'action française, 1925). 
Jamais elle [la mère d'Angéline] n'avait entendu dire qu'un marié se fût conduit de la sorte; mais après y avoir songé, elle se dit qu'il est permis de ne pas agir en tout comme les autres, que l'amour du travail [...]

[...] prenant son rôle de fermière au sérieux, elle alla à sa cuisine [...] L'bistoire des noces fit du bruit, on en fit cent railleries, ce qui amusa fort les auteurs du scandale. ( $A M, 16-17$. C'est moi qui souligne.)

J'ai repris l'habitude de faire lire. [...] L'bistoire me distrait plus efficacement que toutes les autres lectures. Je m'oublie devant ce rapide fleuve des âges qui roule tant de douleurs.

Aujourd'hui j'ai fait lire Garneau [...]

[...]

C'est un de mes regrets de ne l'avoir pas connu [...] son éducation solitaire [...] sans maîtres et presque sans livres, notre historien arrivait à se former.

[...] Enfant [...] Déjà, j'avais le sentiment profond de l'honneur national [...] j'aurais voulu lui assurer la reconnaissance immortelle de tous les Canadiens.

[...] Et nous, Dieu veuille nous donner comme à nos pères, avec le sentiment si français de l'honneur, l'exaltation du dévouement, la folie du sacrifice, qui font les héros et les saints. ( $A M, 134-137$. C'est moi qui souligne.)

Le désir d'Angéline de vouloir "assurer [à l'historien Garneau] la reconnaissance immortelle de tous les Canadiens" incite à conjuguer son goût de toujours pour l'honneur national et le fait que l'histoire la distrait "plus efficacement que toutes les autres lectures". Sans prétendre qu'Angéline de Montbrun soit Laure Conan, on retiendra que l'instance d'énonciation du roman fait que son personnage conjugue spontanément une "valeur ajoutée" à certains textes, la foi religieuse et l'histoire. On peut à tout le moins en conclure que l'abbé Casgrain n'avait pas un point de vue complètement étranger à la pensée de celle qui allait devenir notre première romancière.

À la suite de ce premier travail, en superposant "simplement" - sans analyse systématique - l'anecdote des romans $\dot{A}$ l'ouvre et à l'épreuve, L'oublié et La sève immortelle à celle d'Angéline de Montbrun, j'ai pu suggérer que les quatre récits racontent des histoires dont les vrais héros sont les héroïnes plutôt que les personnages historiques - ou le fiancé qui fait défection? 9 . De manière assez étonnante, en effet, par leur détermination et leurs actions concrètes, les hérö̈nes des trois romans historiques pavent la voie aux [futurs] héros [historiques] ${ }^{10}$; un peu comme si

9. Fernand Roy, "D'Angéline de Montbrun à La sève immortelle: rupture malheureuse ou étonnante continuité?", C. Potvin et J. Williamson (dir.), Women's Writing and The Literary Institution/L'écriture au féminin et l'institution littéraire, University of Alberta, Research Institute for Comparative Literature, 1992.

10. Non seulement la Gisèle Méliand d'Á l'œuvre et à l'épreuve renonce-t-elle sans récriminations à Charles Garnier, son fiancé de toujours, mais elle va jusqu'à obtenir des parents de ce dernier qu'ils acceptent que leur fils aille réaliser son rêve de mourir martyr - elle promet de prendre sa place auprès d'eux aussi longtemps qu'ils vivront. Quant 
le rêve d'enfance prêté à Angéline de mousser la reconnaissance des Canadiens pour l'historien Garneau allait ensuite prendre la forme de romans "humanisant" les héros nationaux.

Au-delà de ces coups de sonde préliminaires, il m'est apparu pertinent de poursuivre le travail et de mener jusqu'à son terme l'étude de l'énonciation des trois romans historiques de Laure Conan. Pour y arriver, je tablerai sur une proposition "sémiotique" qui a été élaborée théoriquement ailleurs ${ }^{11}$. Cette proposition peut être formulée comme suit: un roman argumente en vue de l'attribution (par le lecteur) d'une valeur ajoutée au fait de raconter (par écrit); sa stratégie énonciative consiste à appeler la valorisation de l'histoire - liée à l'écriture - au moyen de la mise en œuvre d'une interaction verbale fictive dont les étapes coïncident avec celles d'une organisation narrative - manipulation, acquisition de compétence, performance et sanction. Je procéderai en deux temps. D'abord, j'établirai par le biais d'une analyse narrative que dans les trois romans les "personnages historiques" sont les destinateurs des héroïnes qui font, elles, figure de sujets de quête. Puis, je montrerai que la stratégie énonciative des trois textes amène subrepticement les lecteurs à assumer de façon imaginaire le rôle de destinateurs - d'abord manipulateurs, puis, finalement, judicateurs - des héroïnes. Avec comme résultat la valorisation de la notion d'histoire que véhiculait l'idéologie de la langue gardienne de la foi.

\section{Des « sujets de quête " féminins manipulés par des «personnages historiques »}

Alors que dans les deux premiers récits le sujet de quête est d'évidence l'héroïne ${ }^{12}$, dans le cas de La sève immortelle, l'identification du sujet de quête fait problème: le personnage historique, Jean de Tilly, est encore vivant à la fin de l'histoire, et c'est également lui qui, d'entrée de jeu, est mis sur le devant de la scène, même s'il y est présenté comme se relevant péniblement d'une blessure très grave subie - assez paradoxalement, on y reviendra - lors de la dernière victoire française contre les Anglais, à Sainte-Foy. Cependant, comme mon objectif est la description

à la jeune Élisabeth Moyen de L'oublié, elle gagne littéralement le droit de soustraire pour un temps Lambert Closse aux tâches militaires, puisqu'elle le sauve d'une mort certaine; mais elle ne tient nullement pour autant à le voir renoncer pour toujours à son métier de soldat de la Vierge. Enfin, la Guillemette de La sève immortelle qu'épousera finalement Jean de Tilly, le héros chargé, au lendemain de la Conquête, de faire durer le souvenir de la France au Canada: c'est elle qui redonne l'espoir à sa future belle-famille en obtenant - d'un riche militaire anglais qu'elle refusera ensuite d'épouser - que leur soient rendus d'abord le matériel nécessaire à ensemencer leurs terres abandonnées durant la guerre, puis le manoir familial.

11. Fernand Roy, "Schéma narratif et énonciation", Protée, vol. XXI, n ${ }^{\circ} 1$, p. 42-49.

12. Les deux personnages historiques avec lesquels elles interagissent sont d'ailleurs morts à la fin des anecdotes. 
de la stratégie énonciative, il m'est nécessaire de commencer par décrire narrativement les trois romans : c'est sur la connaissance des organisations narratives que je grefferai ensuite la question de l'énonciation.

Dans $\grave{A}$ l'œuvre et à l'épreuve, c'est depuis sa tendre enfance que Gisèle Méliand est amoureuse de Charles Garnier, le troisième fils des parents qui l'ont adoptée. L'anecdote débute par sa sortie du couvent de Port-Royal, où elle a été éduquée; sortie qu'elle envisage avec joie, puisqu'elle retrouvera celui qu'elle espère épouser. Elle est cependant profondément intriguée par le discours d'adieu que lui tient la supérieure, en l'occurrence la réformatrice de Port-Royal, l'abbesse Angélique. Celleci lui souhaite non pas le bonheur humain auquel elle aspire, mais que Dieu lui envoie la grâce de connaître "la douleur qui élève l'âme [...] qui arrache aux illusions de la vie" $(C E, 23)$. Une fois revenue chez les Garnier, avant même d'avoir revu Charles dont elle s'explique mal l'absence, elle est carrément manipulée quand, à la vue d'un tableau représentant Ignace de Loyola - la scène se passe dans la chambre de Charles, rien de moins! - sa mère adoptive lui apprend que l'"absent" admire ce tableau parce qu'il lui semble que le saint va dire encore une fois sa célèbre parole: "Ah! que la terre me parait vile, quand je regarde le ciel ${ }^{13} . "(E, 31)$

Toute l'anecdote revient ensuite à ce que Gisèle trouve dans l'écriture d'un journal intime la distance nécessaire (phase de compétence) pour assumer que celui qu'elle aime rêve d'entrer en religion pour devenir missionnaire en Nouvelle-France: Charles ne répondra jamais directement à son amour; tout au plus lui adressera-t-il quelques lettres, une fois qu'elle l'aura aidé à actualiser son rêve. Ces lettres sont disséminées au fil de la narration qui est explicitement donnée comme inspirée des Relations des Jésuites. Dans ces lettres, Gisèle retrouve une traduction on ne peut plus explicite de la sentence d'Ignace de Loyola citée plus haut. Cette lecture rétroactive de la sentence dans un premier temps demeurée énigmatique est rendue possible (phase de performance) par l'action de Gisèle: c'est elle qui a facilité le départ de Charles en acceptant de sacrifier son amour et, même, de pousser l'abnégation jusqu'à convaincre les parents de ce dernier de le laisser partir, en leur promettant de le remplacer en demeurant avec eux. Au terme de la diégèse, la nouvelle de la mort de Charles parvient à Gisèle une fois que, dégagée de sa promesse, elle est devenue carmélite.

Un dernier événement s'avère essentiel à la description de l'organisation narrative du roman. Alors qu'il est plausible que Gisèle soit renseignée au sujet de la vie de Charles en pays de mission - il lui écrit et elle

13. Le lecteur n'est pas complètement insensible au fait qu'Ignace de Loyola prend subrepticement la parole à la place de Charles; s'il est le moindrement plus expérimenté que la jeune Gisèle, évidemment ... 
lit les Relations des Jésuites -, il s'avère par contre plutôt invraisemblable que lui soit rapportée la façon précise dont il meurt : il a choisi, au lieu de fuir les Iroquois comme les autres, de rester complètement seul avec une poignée de Hurons malades qui seront tous massacrés. Il a accepté de mourir pour les aider à mourir chrétiennement, ont rapporté les survivants qui ont fui avant l'ultime attaque. Mais tout se passe comme si Gisèle avait mérité par son abnégation d'en savoir plus que les autres: le frère de Charles, un jésuite devenu provincial des. Carmes, vient lui apprendre que "Charles n'est pas passé par les tourments" $(E, 217)$, que "pendant qu'il [...] préparait [un pauvre Huron] à mourir, 'un Iroquois lui fendit les tempes de deux coups de hache" $(E, 218)$. Rassuree et "relevant sa tête rayonnante", la sœur Gisèle dit alors "qu'il est heureux de ne pas s'être pris au bonheur de la terre" $(E, 219)$. En résumé, Á l'œuvre et à l'épreuve est un récit qui rappelle la vie d'un "saint martyr canadien", Charles Garnier; mais le roman raconte aussi l'élévation progressive, dans la douleur, de l'âme de Gisèle Méliand, qui vient en rendre la réalisation possible. Au plan narratif, le récit de la mort de Charles par un jésuite est essentiellement une sanction de la lecture que s'est donnée Gisèle de la parole initialement empruntée à Ignace de Loyola: il était nécessaire qu'elle ait la confirmation de la pertinence de sa lecture, ce que ne pouvait évidemment faire qu'un autre substitut du fondateur de l'Ordre de Jésus.

L'oublié propose un scénario semblable. La toutè jeune Élisabeth Moyen, dont les parents ont été massacrés par les Iroquois, recouvre sa liberté en échange d'un jeune chef capturé par l'intrépide militaire de carrière qu'est Lambert Closse. Et, malgré son très jeune âge, elle devient immédiatement amoureuse de celui auquel elle doit sa liberté. Pour bien saisir l'enjeu, il faut se rappeler, cependant, que la narration s'ouvre sur une longue mise en situation présentant le fondateur de Ville-Marie, Maisonneuve. Sa petite communauté, vieille de treize années, est dirigée par un "Ordre céleste" qui fait que les militaires qui la protègent ont tous fait le vœu d'être des soldats de la Vierge. En clair: la jeune Élisabeth n'a véritablement pas choisi son lieu pour être amoureuse! Et il n'est pas surprenant, dans un tel contexte de spiritualité, que ses premières larmes d'émotion amoureuse passent, aux yeux de Lambert Closse qui les fait naître, pour des larmes de tristesse: elle est non seulement une pauvre orpheline dont les parents viennent d'être massacrés par les Iroquois, mais elle sera momentanément obligée de vivre dans une petite communauté constamment en butte aux pièges des mêmes hypocrites Iroquois! Cela, Élisabeth ne l'entend pas immédiatement; elle devient tout de suite amoureuse. En ce sens, on peut dire que L'oublié débute par une variante de la situation initiale d'À l'œuvre èt à l'épreuve. Alors que Gisèle entrait symboliquement dans la chambre de celui qu'elle aimait pour y entendre qu'aux yeux de ce dernier la vie terrestre était vide de sens, Élisabeth 
devient amoureuse d'un militaire de carrière qui a promis d'être soldat de la Vierge.

À l'inverse de Gisèle Méliand, Élisabeth acquiert cependant le droit de soustraire pour un temps celui qu'elle aime - et qui l'aime - à son devoir: elle lui sauve la vie ${ }^{14}$. Il accepte alors en toute humilité - étant donné la grandeur de son amour divin et l'importance de sa fonction sociale de substitut de Maisonneuve - la noblesse qu'il y a à vivre un amour humain. Une fois cette compétence acquise, "[l]'amour [ayant] [chez le soldat de la Vierge] triomphé des partis pris surnaturels, héroïques" $(O, 139)$, Élisabeth ne s'attend cependant pas à ce que celui qui a officiellement abandonné son poste de commandant devienne à tout jamais pour elle un simple laboureur. Elle le lui fait savoir assez directement: alors que Lambert Closse l'assure de son amour en lui racontant l'histoire d'un grand seigneur $\mathrm{du} \mathrm{vl}^{\mathrm{e}}$ siècle, heureux d'être devenu un simple moine s'adonnant au dur labeur de cultiver la terre, elle lui répond "plaintivement ${ }^{15}$ " qu'elle "espère" qu'il n'a pas "résolu de l'imiter" $(O, 170)$. Mais l'humilité chrétienne de Lambert Closse ne l'empêchera pas d'être malheureux de ne pouvoir être de l'expédition quand Daulac et les siens iront sauver la colonie entière en donnant leur vie pour contrer le projet des Iroquois: forcer tous les Français à retourner en France ${ }^{16}$. La transformation tient donc à ce que le projet orgueilleux des fondateurs de Ville-Marie réussit, sans pour autant que ceux-ci cessent d'être sensibles à l'amour humain - ce qui n'était pas le cas du missionnaire d'À l'œuvre et à l'épreuve.

Lambert Closse meurt au cours d'une escarmouche et, dans le dernier paragraphe du texte, Maisonneuve se dirige vers sa maison pour annoncer

14. À noter ici qu'en un sens la compétence d'Élisabeth s'apparente à celle de Gisèle: comme celle-ci, celle-là acquiert simplement, par son action, le droit de rester en contact avec celui que son amour risquerait de détourner de son devoir. L'enfant qui naîtra de son union avec Lambert Closse a comme exact équivalent le lien épistolaire que Gisèle a comme privilège de conserver avec Charles Garnier.

15. Élisabeth est toute peinée de le voir "se livrer avec tant d'ardeur à un travail si rudè. Et si Lambert Closse lui raconte l'histoire du moine du $\mathrm{vl}^{\mathrm{c}}$ siècle, c'est pour lui faire apprécier la noblesse du défrichement et de la culture de la terre, une tâche presque aussi belle que celle de "porter le drapeau" $(O, 168)$. Il lui a déjà dit par ailleurs que son rêve serait d'être assez puissant pour la "garder de toute souffrance" et pour la "voir toujours rayonnante" $(O, 165)$. Avant de lire hors contexte et avec nos yeux d'aujourd'hui cette attitude protectrice du soldat, il conviendra de se rappeler que, dès le début de l'histoire, la jeune Élisabeth est déjà étonnante d'autonomie: elle sait déjà "qu'elle lpourrait marcherl sans crainte, sans fatigue, jusqu'au bout du monde, à côté de ce compagnon [Lambert Closse]" $(O, 41)$; et, alors qu'elle pleure d'émotion, tous s'apitoient sur son sort d'orpheline vivant désormais la vie rude des fondateurs de VilleMarie. Pourtant, bientôt, elle n'aura "plus guère de souci de sa sûreté personnelle ", et si les alertes la feront "passer par une sorte d'agonie", ce sera qu'une "autre vie, sans cesse exposée, lui était devenue infiniment plus chère que la sienne" $(O, 82)$.

16. À noter ici un fait important pour l'analyse du roman suivant: Lambert Closse voudrait tellement partir avec Daulac qu'il le supplie, mais en vain, de retarder son expédition suicide après le temps des semences. 
la mauvaise nouvelle à Élisabeth. Pour bien entendre que ce mot de la fin tient lieu de sanction ${ }^{17}$, il est nécessaire d'avoir à l'esprit deux événements: Lambert Closse avait, le soir du Nouvel An, ouvert au hasard un livre saint, pour y chercher un signe annonciateur de l'année qui débutait, et il était tombé sur la phrase suivante, du Livre de Job: "Voilà que je vais m'endormir dans la poussière du tombeau." $(O, 224)$ Pendant que son mari est en train de mourir, Élisabeth s'inquiète d'entendre des coups de feu, mais une Huronne récemment convertie lui demande pourquoi elle craint tant la mort, étant donné que le désir de tout croyant est de se retrouver, enfin, face à Dieu. Ce n'est pas parce que l'on pratique l'humilité qui sied à la condition humaine qu'il convient de subordonner l'amour divin à l'amour humain.

À l'inverse de ce qui se passe dans ces deux romans dont et où la focalisation est largement orientée vers les hérö̈nes, dans La sève immortelle le point de vue de l'instance narrative est presque exclusivement centré sur le personnage historique, Jean de Tilly, et sur le débat de type cornélien que la défaite de 1760 l'oblige à assumer: avant de retourner en France, le chevalier de Lévis lui confie le devoir de faire durer la mémoire française au Canada, alors qu'il aime déjà une noble Française, Thérèse d'Autrée, dont la famille va aussi retourner en France ${ }^{18}$. En temps nor$\mathrm{mal}^{19}$, Jean aurait épousé sa petite cousine, Guillemette de Muy. Mais la défaite annule à tout jamais son espoir de faire une carrière militaire en Nouvelle-France. Les terres de sa famille ont largement souffert des quatre années de guerre et les siens n'ont même plus l'usufruit du manoir familial habité par les troupes victorieuses.

Pour arriver à démêler les fils de cette anecdote plutôt difficile ${ }^{20}$, il est important de bien jauger la portée de son point de départ: Jean de Tilly est en convalescence à la suite d'une [illusoire] dernière victoire militaire qui a failli lui coûter la vie. Si le lecteur commet l'erreur de lier cette blessure à la défaite, il ne s'y retrouve plus parce que, ce faisant, il ignore la symbolique de l'amnésie dont souffre Jean: quand il revient à

17. Maisonneuve y vient confirmer à Gisèle ce qu'elle avait commencé à comprendre, de la réaction initiale de Lambert Closse à ses larmes, en lui sauvant la vie, soit qu'il est bien normal qu'elle pleure, puisque, malgré l'amour humain, elle est appelée à passer sa vie dans un milieu où la mort peut survenir à tout instant.

18. Il convient ici de se rappeler que, dans la mythologie de l'époqué, la Conquête devait constituer une chance providentielle: du coup, le Canada français allait être préservé des méfaits la Révolution française.

19. C'est-à-dire, si ce n'avait pas été de la Conquête: sa mère a en effét déjà accepté que vive chez elle sa cousine Guillemette, qui lui est destinée. Si les choses mettent du temps à reprendre leur cours normal, c'est parce que la guerre a momentanément obligé Jean à revenir à la fonction première de son état: le métier des armes. Jean est en effet d'origine noble!

20. Cette difficulté tient peut-être à ce que Laure Conan en aurait écrit les dernières pages sur son lit de mort. 
lui, il rêve encore d'une carrière militaire en français au Canada. Il convient plutôt de comprendre ${ }^{21}$ que Jean de Tilly est d'entrée de jeu déphasé - d'où son engouement amoureux pour une Française alors que la colonie est en ruine.

Une analyse narrative détaillée du texte conduit alors à mettre en évidence le "féminisme" de notre première écrivaine, qui fait plutôt de Guillemette le sujet de quête de son récit. Celle-ci a secrètement prié pour que celui qu'elle aime en silence ne meure pas de sa blessure, mais voilà qu'à peine remis l'ingrat l'oublie: il ne lui envoie même pas de "belles violettes" puisque leur parfum lui rappelle plutôt Thérèse $(S I, 57)$; il lui achemine de moins passionnés " œillets", en la remerciant amicalement de "n'avoir pas voulu croire qu['il] mourrait" $(S I, 57)$. Cette dernière précision étant apportée, je résume ce qu'il convient de retenir.

1) En pratique, Thérèse est l'anti-sujet de la quête de Guillemette. Une lettre d'invitation écrite de sa main par son père également blessé redonne à Jean espoir en la vie qu'il espérait en un autre temps: il se rappelle avoir déjà remarqué Guillemette lors d'un défilé militaire, après la victoire de Carillon. Et, élément significatif, une fois amoureux de Thérèse, lorsque son frère aîné vient lui parler des siens (et de Guillemette), lui revient le rêve qu'il a fait au sujet de l'enterrement de Montcalm: "Quand on prit le cercueil pour le mettre en terre, je saisis le drapeau [...] je voulus le rouler, mais le drapeau m'échappa des mains s'éleva très haut [...] s'étendit au loin $[. .$.$] couvrit la terre canadienne." (S I, 52)$

2) La manipulation porte cependant sur l'héroïne Guillemette. Fille unique et orpheline de mère, elle habite avec la famille de Jean parce que son père $a$, après la victoire de Sainte-Foy, suivi l'armée française vers Montréal. Elle se retrouve en toute lucidité dans une situation on ne peut plus contradictoire: elle fait profiter la famille des Le Gardeur de Tilly de l'intérêt que porte à sa personne un riche militaire anglais, alors qu'en retour sa présence dans cette famille embarrasse Jean.

3) Une fois les hostilités terminées, avant de retourner en France, Lévis, vainqueur de Sainte-Foy, confie à Jean la mission de faire durer le souvenir de son pays en terre canadienne. Ce qui finit de placer Jean dans un dilemme cornélien, puisqu'il a eu, juste avant, la confirmation que Thérèse l'aime aussi. Disons-le tout net: depuis sa blessure, Jean n'arrive plus à vivre au présent et son égarement amoureux immédiat en fait le destinateur-manipulateur de Guillemette. En femme qui a toute sa tête ${ }^{22}$, et malgré la gêne de Jean à son endroit, Guillemette refuse la

21. Gilles Marcotte a bien senti la chose, qui a écrit que Laure Conan "enrichit sa vision de l'histoire d'une expérience toute personnelle de la misère ": notre première romancière semble projeter une image masculine de la réussite.

22. En cela, elle est comme la mère de Jean. Je serais porté à dire que c'est toujours comme cela dans les romans de Laure Conan et que c'est peut-être ce qui pourrait 
proposition de mariage du riche militaire Laycraft, proposition que son propre père lui suggère d'accepter, puisque le prétendant lui garantit qu'elle pourra conserver sa foi. En prenant ainsi position, elle ouvre une troisième voie, entre le rêve militaire de Jean qui le conduirait à faire carrière en France et la désespérance de son père, militaire également, pour qui c'est une chimère que d'imaginer qu'il y ait encore de l'avenir pour le français au Canada: "J'espère que sur la terre canadienne, la semence française vivra", répond en toute simplicité Guillemette quand le prétendant anglais lui demande ce qu'elle espère en refusant de quitter sa vie de misère. Le choix de Guillemette la qualifie pour la suité de sa quête.

4) La performance ne tient à Jean qu'en apparence; concrètement, il s'avère qu'il est plutôt agi. Sa "blessure de la victoire de Sainte-Foy" s'ouvre de nouveau - symboliquement cette fois - quand, venu annoncer à sa mère sa décision d'aller s'installer en France avec celle qu'il aime pour vivre de son métier de militaire, il finit par se rendre aux arguments maternels (honneur et devoir envers la patrie) et décide de rester au pays. Cette réouverture de la blessure tient d'évidence lieu de transformation de sa situation de départ - plus spécifiquement la scène du chapitre IV où son frère vient lui donner des nouvelles de Guillemette et au cours de laquelle il lui envoie des "œillets" plutôt que de "douces fleurs", des violettes. Il convient de comparer les circonstances des deux blessures. La blessure survient encore lors d'une victoire en terre canadienne. Mais cette fois, la victoire est remportée par une femme - la mère, qui veut voir son fils épouser Guillemette - et elle est suivie de l'abandon de la France par le Canada, alors que, au lendemain de la victoire de SainteFoy, c'était la France qui avait abandonné sa colonie. Cette différence mérite toute notre attention: le lendemain, en effet, un bateau anglais arrive à Québec, comme cela avait été le cas après les événements de Sainte-Foy, mais le bateau n'apporte pas des renforts, il vient au contraire prendre la famille de Thérèse et part avec elle vers l'Angleterre, libérant du coup les Gardeur de Tilly de toute lubie de noblesse à la française. Guillemette triomphe, non par les armes, mais plutôt symboliquement: elle méritait bien un coup de main de la part de la mère de Jean, puisque sans elle les Gardeur de Tilly n'auraient pas de sitôt recommencé à cultiver leurs terres et à vivre dans leur manoir.

5) Thérèse meurt peu après son arrivée en France; mais avant de mourir, elle aura, dans une seconde lettre, assuré Jean de l'indéfectibilité de son amour: "Si mon heure est venue, comme je le crois, ne vous

mener à nuancer un jour le jugement de Marcotte, jusqu'à parler non pas tant de la place de la misère dans la vision de l'histoire de Laure Conan, mais bien de l'expérience d'une femme qui n'a jamais adhéré à une vision positiviste de l'histoire. Il y aurait là, peut-être, matière à saisir une fois pour toutes la raison profonde du différend entre elle et Casgrain. 
désolez pas, ne me confondez pas avec mon cadavre. Je vous aimerai tant que mon âme vivra." ( $S I, 228) \mathrm{Et}$, un an après, Guillemette de répondre un "Si je le veux! " d'une "ravissante simplicité" $(S I, 231)$ à Jean lui demandant si elle veut être sa femme.

Maurice Lemire avait vu juste au sujet de l'anecdote des romans historiques de Laure Conan: les trois sont des variantes de celle d'Angéline de Montbrun. Il convient cependant de remarquer que ces variantes ne sont pas que de simples répétitions. D'un texte à l'autre, en effet, il y a évolution vers un rapprochement final de l'héroïne et de son destinateur, le personnage historique. À la fin d'Angéline de Montbrun, dans le médaillon qu'elle porte jour et nuit et où se côtoyaient les photos de son père et de son fiancé, ce dernier se voit remplacé par une médaille de la Vierge. À la fin d'À l'oeuvre et à l'épreuve, Gisèle a acquis la conviction qu'elle retrouvera celui qu'elle aime au ciel. Dans L'oublié, Élisabeth donne naissance à un enfant avant que ne meure Lambert Closse. Et $L a$ sève immortelle se termine par une demande en mariage. On doit bien sûr souligner que dans ce contexte la sexualité est devenue un simple devoir de procréation et que, dans le troisième roman, l'amour humain ne va pas sans mauvaise conscience. Pourtant, il me paraît utile et nécessaire de dépasser ce niveau d'interprétation, qui homologuerait texte et désir sexuel, pour se rappeler avec Freud que la culture résulte du refoulement de la sexualité.

Au terme de ce travail sur l'organisation narrative des trois romans historiques, il me semble pertinent de souligner que chacune des anecdotes des romans de Laure Conan tend en elle-même à corriger partiellement une interrogation "matérielle" en la mettant dialectiquement en rapport avec une problématique "spirituelle". Dans tous les cas, l'interrogation spirituelle est celle du sens de l'existence au-delà de la vie que je traduirai en termes de signifié pensé comme éternel - et la problématique matérielle est celle de l'amour humain - que je traduirai en termes de signifiant à rendre opérationnel. Dans tous les cas également, la problématique anecdotique est celle de l'instabilité des relations amoureuses; celle-ci, après avoir été identifiée comme mineure, permet d'apporter de l'espoir.

Chaque fois, comme je l'ai donné à entendre au départ en soulignant la fonction de l'Histoire de Garneau dans Angéline de Montbrun, c'est un discours à caractère historique qui permet la transformation de la problématique amoureuse. Les lettres de Charles, étayées et parfois même citées par les Relations des Jésuites, sont données à lire à Gisèle au fil de la diégèse d'À l'ouvre et à l'épreuve et, aux yeux de cette dernière, ces documents constituent une incitation à se faire carmélite pour être digne de l'amour de Charles dans l'au-delà. L'histoire du grand seigneur du $\mathrm{vI}^{\mathrm{e}}$ siècle devenu moine et vivant heureux à cultiver humblement la terre, que raconte Lambert Closse à Élisabeth pour la convaincre de son amour, 
constitue, aux yeux de cette dernière, une preuve qu'un "soldat de la Vierge" demeure un humain malgré sa mission divine. Comme en fait foi la réponse d'Élisabeth, il ne saurait cependant être question d'oublier le provisoire pour l'essentiel: être pleinement humain et s'accepter comme tel ne fait pas nécessairement oublier l'essentiel, le sens de l'existence! Et le revirement soudain de Jean de Tilly, dans La sève immortelle, tient essentiellement à ce que sa mère sait lui fournir les arguments historiques qui lui manquaient pour qu'il continue de croire à la civilisation française au Canada; elle lui rappelle en effet des paroles de Marie de l'Incarnation: "Le Canada est un pays spécialement gardé par la Providence." (SI, 209) Si Jean se rend à ses arguments, c'est parce qu'il sait que ces paroles ont été vérifiées par la vie même de Marie de l'Incarnation. Comme le savoir historique' est, dans les quatre romans, posé comme authentique, on peut se demander s'il n'en résulte pas, comme effet d'ensemble, que la langue française actualisée dans les fictions finit par s'avérer gardienne de la foi, c'est-à-dire du sens possible qu'elle permet de construire. C'est une hypothèse qui gagnera évidemment à être étayée par une analyse de la stratégie énonciative mise en place dans chacun des romans historiques de Laure Conan.

Entre le premier et le troisième roman, en effet, il n'y a que dans le troisième qu'un enfant naît avant que ne soient finalement réunies, sur le modèle du médaillon du premier, la figure paternelle de Maisonneuve et celle d'une mère humaine. Et entre le second et le quatrième, il n'y a que dans le quatrième que la garantie d'un amour éternel rend possible la procréation - alors que c'était le contraire dans le second. De la sorte, $L a$ sève immortelle peut être lue comme une sanction faisant retour sur L'oublié (comme performance) validant, eu égard à la problématique mise en place dans Angéline de Montbrun, la compétence acquise dans À l'œuvre et à l'épreuve. Dès lors, on en arrive à formuler l'idée que les quatre romans de Laure Conan mettent en place l'idée que la langue française est gardienne de la foi - comme le donne à lire la performance du dernier roman, qui renvoie d'autant plus allègrement vers l'Europe la noblesse française que ce sera bientôt là-bas la Révolution. En motivant le mariage en fonction du contexte historique québécois, les romans construisent une cohérence qui actualise une vision de l'histoire associée à l'idéologie selon laquelle la langue française est gardienne de la foi catholique.

\section{L'instance d'énonciation dans les romans « historiques "}

En 1902, dans sa préface à la seconde édition de L'oublié, l'abbé G. Bourassa écrivait ceci, qui me semble une bonne piste pour aborder l'étude de la stratégie énonciative des trois romans historiques de Laure Conan:

Mais notre devoir, étant donné le héros du livre et son milieu historique, tel que nous le décrivent les documents contemporains, est de louer hautement 
l'auteur d'avoir si fidèlement attribué au major de Ville-Marie, ainsi qu'aux autres personnages de son livre, l'esprit et les sentiments qui furent en réalité les leurs.

Son délicat et pur roman - qui n'est peut-être pas, à vrai dire, absolument un roman - y trouve le mérite d'être, historiquement, plus vrai, comme il emprunte aux souvenirs héroïques et pieux qu'il évoque un parfum de poésie chevaleresque et mystique, qui a fait dire à un écrivain rationaliste racontant, en historien simplement, la naissance de Montréal: "Est-ce de l'histoire vraie, ou n'est-ce pas plutôt un roman de chevalerie chrétienne? C'est l'un et l'autre ${ }^{23}$."

Pour rendre compte dudit "mérite d'être, historiquement, plus vrai" qui résulterait de la stratégie consistant à reproduire l'esprit et les sentiments des personnages historiques tout en imaginant par ailleurs ceux des autres personnages dont on sait moins de choses, j'ai commencé par établir que les personnages historiques représentés dans ces romans ne sont pas les sujets de quête des anecdotes dans lesquelles ils "revivent". Il n'aurait pas été suffisant de reconnaître qu'un personnage romanesque, fût-il historique, n'est jamais qu'un personnage de papier; ce simple constat ne permet pas de concevoir clairement que la stratégie énonciative de Laure Conan a consisté à valider les faits et gestes connus des personnages historiques par ceux, forcément imaginés, des sujets de quête qu'elle imagine. C'est une chose qu'elle ait cru à ce que l'historien Garneau a raconté au sujet de Daulac, et c'en est une autre qu'elle ait profité de ce que Lambert Closse avait été négligé par les historiens pour faire d'Élisabeth Moyen l'héroïne de son roman. Dans cette perspective, je m'appliquerai à montrer que les anecdotes des trois romans historiques structurent des simulacres d'interaction verbale entre les personnages historiques auxquels renvoient ses titres et des hérö̈nes imaginaires, interactions qui amènent les lecteurs à occuper le rôle des destinateurs des anecdotes.

Au début d'À l'œuvre et à l'épreuve, Gisèle de Méliand devient sujet de quête à la suite de deux échanges avec des personnages historiques qui lui tiennent des propos "manipulateurs": le propos de la mère Angélique, l'abbesse de Port-Royal, lui souhaitant la grâce de connaître "la douleur qui élève l'âme", est repris dans celui d'Ignace de Loyola, le fondateur de l'Ordre de Jésus. En envisageant cette manipulation sur le plan de l'énonciation, on constate que, d'une "parole" à l'autre, il y a un important changement. Premièrement, lors de la seconde, l'auteur de la "sentence", dont le contenu éloigne Gisèle de son projet amoureux, est représenté par un tableau : la scène entre la mère de Charles et Gisèle se passe devant un portrait d'Ignace de Loyola. Deuxièmement, la seconde

23. G. Bourassa citant Francis Parkman (The Jesuits in North America in the Seventeenth Century, tome 2, Frontenac Édition, Toronto, Morang, 1900, p. 25). 
"sentence" donnée comme célèbre est présentée à Gisèle comme étant souvent répétée par Charles qui, à ce moment, est absent. Troisièmement, l'interlocutrice de Gisèle n'est pas en l'occurrence un personnage historique connu, et ce personnage se situe carrément du côté du quotidien: selon son fils, "il semble que le saint va dire encore: "Ah! que la terre me parait vile, quand je regarde le ciel." " $(E, 31)$

Si l'amoureux que Gisèle espérait est absent le jour du retour de cette dernière du couvent, par contre sa place est tenue par un "il " à la parole duquel il s'identifie. De telle sorte que Gisèle n'est littéralement pas en mesure, dans l'immédiat, de réagir au propos qu'ón lui dit être celui de Charles! Au plan énonciatif, il y a bel et bien un problème: en revenant chez ses parents adoptifs, Gisèle espérait trouver en Charles un amoureux, et voilà qu'au lieu de se retrouver en face de son "interlocuteur", elle reçoit indirectement une parole empruntée à un "il" et qui suggère que l'amour humain paraît bien vil quand on regarde le ciel. Gisèle' se retrouve dans l'impossibilité de réagir en adoptant la symbolique, que semblent partager tant la mère Angélique que le célèbre fondateur de l'Ordre de Jésus.

Elle se tiendrait en face de Charles un verre d'eau à la main et Charles lui dirait que c'est du vin qu'il veut, que ce serait un moindre mal. Sa quête consisterait alors à assumer la demande de Charles, à remplacer l'eau par du vin. Dans l'immédiat, la demande de Charles est plutôt de l'ordre d'un "c'est bien de l'eau que je veux, mais sans verre!". Par contre, son absence ouvre une possibilité différente: en remplaçant le locuteur par une parole "historique", l'instance d'énonciation amène Gisèle à vouloir comprendre la sentence. Littéralement, celle-ci est manipulée par une situation d'"écriture": c'est la piste qui lui est fournie pour atteindre Charles.

À la toute fin, c'est un jésuite, et, partant, un autre substitut d'Ignace de Loyola, qui vient raconter à sœur Gisèle les circonstances de la mort de Charles. Du coup, sour Gisèle tient enfin la confirmation de la pertinence de la lecture qui l'a transformée elle-même au fil de la diégèse. J'insiste: le récit de la mort de Charles ne doit pas être confondu avec la nouvelle même de cette mort que possède déjà sœur Gisèle, par le biais de la lecture des Relations. Ce récit oral renvoie plutôt à la problématique soulevée par la conversation initiale entre l'abbesse et la jeune femme: celle-ci allait quitter le couvent, alors que maintenant elle a renoncé au monde. Le jésuite tient maintenant la position mondaine initialement occupée par Gisèle; et celle-ci élève la discussion à un niveau de "haute spiritualitén, comme le faisait l'abbesse. L'essentiel de la scène n'est pas dans ce que le jésuite raconte, mais dans la réaction, l'interprétation, de son interlocutrice:

Le monde extérieur avait complètement disparu pour elle [...] Et, tout à coup, emportée vers le passé, elle le revit tel qu'elle l'avait vu, sur les bords de la falaise, en face de l'océan, alors qu'il luttait contre son amour. 
"Ah! mon Père, dit-elle, répondant à sa [propre] pensée, et relevant sa tête rayonnante, qu'il est heureux de ne pas s'être pris au bonheur de la terre [...]" $(C E, 218-219)$.

Je reviendrai à cet épisode de Charles "luttant", imagine Gisèle, contre son amour pour elle; d'abord, il convient de noter que, du récit $d u$ jésuite, elle retient uniquement ce qui tient lieu de sanction de sa propre bypothèse au sujet de l'attitude de Charles à son endroit. Elle réussit en quelque sorte la dernière épreuve qui la rend aussi spirituelle que l'abbesse. Elle peut désormais relever "sa tête rayonnante": dans l'au-delà, elle sera digne d'être aimée éternellement par lui.

Entre la manipulation et la sanction qui est le fait de l'Ordre de Jésus, Gisèle a posé (l'écriture intime aidant) une hypothèse au sujet de l'énigmatique silence de Charles regardant l'océan où le sieur de Champlain allait s'embarquer pour retourner au Canada sans son épouse qu'il aimait pourtant beaucoup. Elle a imaginé que s'il ne répondait pas à son amour, c'était moins parce qu'il ne l'aimait pas que parce qu'elle lui inspirait un amour venu de l'au-delà. Elle a en somme inventé une manière de décoder pour elle-même la sentence de Loyola. Envisagée dans une perspective énonciative, la scène du départ de Champlain est intéressante: cette fois en présence de Charles, mais à son insu, par le biais d'un journal intime, Gisèle se donne de lui une image qui peut être conciliée avec la sentence de Loyola. À l'inverse de ce qui s'était passé en face du portrait de ce dernier, cette fois elle crée un sens possible à l'attitude de Charles à son endroit. Ce n'est pas rien: du coup, Gisèle assume dans l'écriture le silence de Charles; l'écriture est d'ailleurs la seule forme d'expression qu'il empruntera désormais pour lui parler "de l'autre bout du monde". L'acquisition d'une compétence de lecture au moyen de l'écriture intime donne à Gisèle l'espoir de devenir ensuite une lectrice adéquate de la socialisation de la parole empruntée par Charles à Loyola.

Dans le troisième temps de l'anecdote, Charles est missionnaire au Canada et il écrit à Gisèle quelques lettres un peu "personnelles". Parallèlement, d'autres nouvelles de Charles parviennent à la jeune fille par le détour des Relations. Là stratégie est intéressante: les lettres personnelles facilitent la compréhension des Relations et font de Gisèle une lectrice désormais avertie des adeptes de la Société de Jésus. L'important pour mon propos est de remarquer que ce sont ces lectures qui la transforment en carmélite. Par elles, se voit actualisé l'idéal que lui fixait initialement la mère Angélique quand elle lui parlait de la douleur qui purifie: c'est dans les Relations qu'elle apprend la mort de Charles et qu'elle expérimente la validité de la sentence d'Ignace de Loyola.

Il fallait pourtant, pour que le simulacre d'interaction soit complété, que l'on revienne ensuite à l'oral, au récit final du jésuite, au cours duquel sœur Gisèle assume finalement la parole de la mère Angélique. 
De toute évidence, l'énonciation de L'oublié répond à une stratégie équivalente, encore que tissée plus finement. Très impressionnée par celui que Maisonneuve désigne pour la reconduire à sa future demeure, soit son sauveteur Lambert Closse, la jeune Élisabeth Moyen lui dit timidement la naissance de son amour par le moyen de larmes d'émotion qui lui échappent, larmes que. son sauveteur lit évidemment comme une "demande de protection" que lui adresse une pauvre petite orpheline qui n'a "en apparence" pas la détermination chrétienne nécesssaire pour vivre les débuts héroïques de la fondation de Ville-Marie. En l'occurrence, c'est celle qui interrompt la conversation entre Lambert Closse et Élisabeth, soit Jeanne Mance, l'" héroöne de Ville-Marie " $(O, 43)$ qui tient lieu d'antisujet $^{24}$.

Tous sont cependant bientôt impressionnés par la force de caractère exceptionnelle dont fait preuve de la jeune Élisabeth; on 's'en étonne même dans les lettres que s'échangent entre elles les religieuses de Montréal et de Québec. Il convient, encore ici, d'insister : il est question de l'attitude incompréhensible de la jeune femme dans des écrits. Puis, un bon soir, voilà que Lambert Closse perd sa légendaire impassibilité : "Il ouvrit un livre, mais l'image d'Élisabeth était restée dans ses yeux." $(O, 114)$ C'est par le biais de la lecture qu'Élisabeth s'infiltre dans la tête de son interlocuteur. La petite Moyen - c'est son nom! - acquiert en cette occasion la capacité de lire par elle-même l'ordre symbolique qui l'empêchait initialement de communiquer ses sentiments humains à un soldat de la Vierge le soir de son arrivée ${ }^{25}$. Cette compétence, elle l'exercera par la suite d'une manière tout aussi inattendue. Mais avant, il est utile de souligner que cette scène de lecture retourne en son contraire la réponse de Lambert Closse aux larmes de la jeune femme: cette fois, ce sont les yeux de Lambert Closse qui "pleurent" d'eux-mêmes, faisant apparaître sur les pages d'un livre de spiritualité l'amour humain que sa réponse initiale voulait orgueilleusement ignorer. Dès lors, Élisabeth peut participer à la mission des héros de Ville-Marie. À son tour, elle sauve la vie de Lambert Closse! Tant et si bien que, momentanément, "l'amour avait triomphé des partis pris surnaturels, hérö̈ques" $(O, 139)$. Conclusion temporaire: alors qu'initialement il n'y avait pas de place à Ville-Marie pour l'amour humain, voilà que maintenant, une lecture inattendue aidant, Élisabeth peut dialoguer d'égal à égal — ou presque - avec un soldat de la Vierge.

24. Je ne sais réellement pas quelle conclusion tirer de la chose, mais, d'évidence, tous les romans historiques de Laure Conan proposent une vision douce de l'utilisation de la violence. Est-ce une caractéristique à porter au crédit de la féminité?

25. En s'apercevant qu'elle pleurait, il lui avait dit d'un ton protecteur et paternel: "Pauvre enfant! [...] il faut avoir du courage. Puis qui sait ce que l'avenir nous réserve... j'ai vu de beaux jours qui avaient commencé par d'affreux nuages." $(O, 42)$ Il y a tout de même moyen de jouer sur le "ce que l'avenir nous réserve...." 
Une fois acquise la compétence qui lui manquait pour avoir le droit d'être aimée humainement à Ville-Marie, Élisabeth lit comme il convient, en fonction de la mission divine poursuivie par cette collectivité, l'histoire du noble chevalier du $\mathrm{vI}^{\mathrm{e}}$ siècle devenu moine et simple laboureur que lui raconte son mari pour l'assurer de son amour: elle ne s'attend pas à ce qu'il devienne une pâle imitation d'un moine du vi ${ }^{\mathrm{e}}$ siècle ${ }^{26}$ ! Son point de vue à elle s'inscrit ainsi dans l'ordre symbolique qui lui était étranger au point de départ; elle refait (du personnage historique) de Lambert Closse un soldat de la Vierge, mais un soldat qui sait maintenant éprouver des sentiments humains. La transformation est accomplie, la réponse d'élisabeth ramène à sa mission spirituelle de héros historique celui qu'elle aime, alors qu'initialement son amour risquait de l'en détourner.

Quand le roman se termine avec la scène où l'on voit Maisonneuve s'acheminant vers la résidence du couple pour annoncer à Élisabeth la mort de Lambert Closse, la boucle est à peu près bouclée. Au départ, Élisabeth n'avait pas eu le temps de répondre aux mots de consolation de Lambert Closse, puisque Jeanne Mance avait, par hasard, fait cesser le début de dialogue intime en ouvrant la porte - elle les avait vus venir! À la fin, ce que Maisonneuve lui dira n'est pas non plus écrit; au lecteur d'assumer qu'Élisabeth occupe désormais une position comparable à celle de Jeanne Mance au point de départ. À lui d'imaginer qu'Élisabeth saura réagir, alors qu'elle vient tout juste d'accepter, lors d'une conversation avec une nouvelle croyante, qu'un chrétien ne doit pas avoir peur de la mort, puisque celle-ci lui permet de voir enfin Dieu.

On commence à mieux sentir la stratégie énonciative qui gouverne $L a$ sève immortelle quand on remarque que c'est la main d'écriture ${ }^{27} \mathrm{de}$ Thérèse d'Autrée qui impressionne Jean de Tilly, et pas du tout le contenu de l'invitation de son père qu'elle est chargée de lui transmettre. Est-il plus opératoire stratégie, pour pointer la substance de l'expression - en l'occurrence l'écriture - d'un message, que de le faire lire par un homme qui s'arrête à la finesse et à l'élégance de l'écriture, se disant qu'il s'agit sans doute d'une main de femme? Ce qui est moins évident, par contre, c'est en quoi cette main d'écriture fait que le personnage historique qu'est Jean de Tilly s'avère le destinateur-manipulateur de Guillemette.

À la fin de l'histoire, la même main d'écriture revient, sans que cette fois on en apprenne autre chose que le contenu: sur son lit de mort,

26. Il y a cependant lieu de noter que, tout au long de la première partie du roman, cette allusion à un noble du $\mathrm{vi}^{\mathrm{c}}$ siècle devenu simple laboureur était annoncée par le biais d'une comparaison entre la situation de Ville-Marie et un village du même siècle.

27. "Monsieur de Tilly regarda l'adresse. L'écriture fine, élégante, lui était inconnue et lui parut d'une femme." (SI, 28); "Plusieurs fois, il relut la lettre cherchant à deviner ce que Mademoiselle d'Autrée pensait en écrivant ces lignes [...] il lui semblait qu'une vive sympathie s'en dégageait, et il avait envie de baiser la signature; "Thérère d'Autrée". " $(S I, 31)$ 
Thérèse insiste pour que Jean oublie son cadavre, mais se souvienne que son âme l'aimera tant qu'elle vivra. De la finesse et de l'élégance d'une main (française) d'écriture, à l'amour de l'âme de cette même (main) française à faire durer par le souvenir au-delà de la matière, est-il plus efficace façon de donner un esprit - une forme - au contenu d'une lettre que d'en assurer la pérennité par le souvenir? Bien que sensible à la stratégie de la main d'écriture, le lecteur aura cependant un mouvement de recul: l'invite-t-on à entendre que Jean va épouser Guillemette par devoir, tout en continuant d'aimer l'âme de Thérèse?

Il convient plutôt d'envisager la question dans une perspective de mise en œuvre d'un simulacre d'interaction dont Jean est le locuteur et Guillemette l'interlocutrice. Ce qui rend la quête de Guillemette problématique, c'est que celle-ci pratique un mode d'expression qui à première vue ne parait guère adéquat pour assurer le souvenir de la France en terre canadienne: elle semble être prête à pactiser quotidiennement avec les Anglais, allant même jusqu'à en laisser un cueillir pour elle les fleurs du jardin de la famille de Jean. Disons la chose autrement : elle est assez "charmante" physiquement pour attirer l'attention d'un riche Anglais la voyant travailler aux champs ${ }^{28}$, mais, au point de départ, elle ne semble pas être en mesure de répondre adéquatement aux aspirations spirituelles d'un militaire de carrière chargé, pour reprendre cette expression, de la survie du souvenir de la France en terre canadienne.

La situation commence à évoluer le jour où Guillemette, sans faire usage de la substance d'expression qu'est l'écriture - elle n'a pas une aussi "belle" main d'écriture - , actualise de mémoire une parole que la célèbre éducatrice Marie de l'Incarnation avait servie à un noble étonné de son attitude à la suite de l'incendie de son couvent. Cette parole lui sert d'argument pour expliquer au riche prétendant anglais que, pour réaliser son idéal, elle n'a pas à désespérer du présent. En l'occurrence, le "Monsieur de Repentigny, pourquoi ne serions-nous pas contentes, nous savons que Dieu est notre père!", qu'elle cite de mémoire, la qualifie comme interlocutrice éventuelle de Jean, à qui a été confiée la lourde et en apparence impossible - mission que l'on sait: elle motive sa sérénité en faisant valoir que Marie de l'Incarnation a eu raison d'avoir confiance en son Père éternel, puisque son entreprise s'est effectivement révélée florissante par la suite. Ce n'est pas là une "parole belle mais vaine sur le plan du sens à venir": elle est fondée sur des événements vérifiés! Du coup, on l'entend, Guillemette se révèle capable de faire des lectures pertinentes, et à plus long terme que ne le laissait croire son seul charme physique immédiat.

28. On aura fait le lien avec l'attitude de Lambert Closse essayant d'imiter un humble cultivateur de la terre. 
Le point tournant de l'anecdote tient, on se le rappelle, à ce que madame de Tilly réussit à infléchir la décision de son fils d'aller vivre en France. À cette occasion encore, c'est une parole de Marie de l'Incarnation qui tient lieu d'argument décisif: "Le Canada est un pays spécialement gardé par la Providence. " Cependant, il ne s'agit plus seulement d'un "nous" intimiste, valant uniquement pour quelques femmes et un hôpital, mais bien du Canada tout entier! Et, cette fois encore, la parole est donnée comme vérifiée historiquement. La première blessure de Jean lui avait somme toute été heureuse, puisqu'elle lui avait donné l'occasion de découvrir une "main d'écriture" qui allait lui redonner goût à la vie; en définitive, la seconde blessure lui est également heureuse, puisqu'elle sera pour lui, avec le temps, l'occasion de reprendre espoir pour la survie du souvenir français au Canada. Et cette seconde fois constitue la transformation de la situation initiale de Guillemette: du coup, elle cesse d'être une simple charmeuse d'Anglais pour s'avérer une interlocutrice de haut niveau pour Jean.

C'est ce qui motive, je pense, son "Si je le veux" final (je souligne): sa réponse à la demande en mariage que se décide finalement à lui adresser Jean de Tilly n'est pas à lire à un premier niveau, mais eu égard au jeu qu'elle a joué et qui a consisté, "énonciativement parlant", à se donner la compétence de lectrice qui ne lui était pas acquise au départ, au temps où Jean la remerciait amicalement de quelques fleurs parce qu'elle avait prié pour qu'il ne meure pas. Bien sûr, à ce moment-là, ses intentions n'avaient pas encore été vivifiées par l'écriture - par le souvenir des paroles "à l'inspiration vérifiée" de Marie de l'Incarnation.

\section{En guise de conclusion}

Convient-il d'avoir l'humilité intellectuelle de reconnaître que les théories littéraires, généralement élaborées sans prendre en compte la situation d'écriture qu'elles permettent d'exploiter de "manière libérale", souffrent du même idéalisme que l'idéologie cléricalo-religieuse de la langue française gardienne de la foi qui a prévalu au Canada français avant l'avènement de la littérature québécoise? Depuis 1960, s'est élaborée au Québec une idéologie laïque d'un pays à construire d'abord dans les mots. À mon sens, cette croyance dans l'écriture est un équivalent laïque de la foi chrétienne dans l'idéologie cléricalo-religieuse que les romans historiques de Laure Conan ont, en leur temps, contribué à rendre opératoire. De la littérature canadienne-française comme constitution du patrimoine sacré de la langue française à la littérature québécoise comme subversion libératrice des forces vives de la collectivité, ce qui aurait changé ne serait pas l'écriture littéraire mais bien le rapport à l'histoire de cette écriture. Du "patrimoine sacré" au "pays à inventer", il y aurait simplement prise de conscience de ce que l'histoire est à faire - et affaire d'écriture. Prétendre que les textes littéraires sont modernes et 
valables quand ils participent de la subversion de l'ordre établi, ce serait encore faire preuve d'idéalisme, ce serait encore méconnaittre que l'ordre en question continue d'être celui de l'écriture.

La description de la stratégie énonciative des romans de Laure Conan m'aura servi de point d'appui pour objectiver un des interdits qui rendent possible notre vision du monde fondée sur l'écriture, soit l'impossibilité de rabattre l'une sur l'autre la littérarité et l'historicité. La littérature comme phénomène social tiendrait sa spécificité de ce qu'elle rend possible l'histoire, en mettant en place un rapport de contradiction entre le plan de l'expression et le plan du contenu des langues écrites. En montrant que les romans historiques de Laure Conan valorisent l'historicité en proposant des fictions d'interactions verbales où des figures d'écriture prêtées à des héros sont décodées sans reste par des hérö̈nes au moyen d'autres figures d'écriture, j'espère avoir un peu donné à comprendre qu'ils relèvent de notre préhistoire parce qu'ils faisaient une équation d'identité entre littérarité et historicité. Il me paraitrait tout aussi regrettable d'essayer en retour de faire une équation d'identité entre historicité et littérarité. Il y a évidemment un reste: une sémiotique fondée sur la notion d'interaction verbale. Puisse ce reste non pas donner à penser qu'il ne sert à rien de construire des histoires littéraires, mais bien aider à assumer intellectuellement que la notion d'histoire est aussi, comme toutes les significations liées à l'écriture, le résultat de fictions d'interactions verbales. C'est Freud qui, si j'ai bonne mémoire, disait qu'à prendre le rêve pour la réalité on risque toujours de finir par perdre de vue la réalité même.

En décrivant la stratégie énonciative des romans historiques de Laure Conan, je n'avais en somme qu'une prétention: donner à entendre que si le fait de raconter des histoires semble remonter à l'origine de l'humanité, cela tient sans doute à ce qu'en soi une signification est effective, au-delà de l'interaction qui la génère, quand deux locuteurs sont en mesure de la raconter ensemble, en alternant les rôles, c'est-à-dire en tenant soit le rôle de locuteur (manipulation et sanction), soit le rôle d'interlocuteur (compétence et performance). Avec comme conséquence que la notion de fiction, entendue au sens de capacité, et donc de compétence, de tenir seule les quatre rôles, serait la condition nécessaire à l'histoire, la manière de rendre compte du réel de façon opérationnelle et donc de performance, c'est-à-dire par définition à jamais en manque de sanction définitive. 